\title{
Editorial zum Themenschwerpunkt Vorfußchirurgie
}

Die Vorfußchirurgie hat in den letzten 20 Jahren einen grundlegenden Wandel erfahren. Die über viele Jahrzehnte großzügig indizierten Resektionsarthroplastiken zur Stellungskorrektur des Großzehengrundgelenks sind weitgehend zugunsten einer rekonstruktiven Chirurgie verlassen worden. Dabei ist es gerade aus historischer Sicht unverständlich, warum sich die Resektionsarthroplastik am Großzehengrundgelenk, die sog. Keller-Brandes-Operation, trotz ihrer offensichtlichen Nachteile so lange als Standardoperation der Hallux-valgus-Deformität etabliert hatte. So traten Karl Ludloff (1918) und Georg Hohmann (1925) schon vor nahezu 100 Jahren engagiert für eine gelenkerhaltende Behandlung des Hallux valgus ein. Auch die von Georg Hohmann formulierten Ziele einer Spreizfußkorrektur mit Hallux-valgus-Fehlstellung, nämlich

- Beseitigung des Schiefstands der Großzehe,

- Korrektur der pathologischen Abspreizung des Metatarsale I,

-Wiederherstellung des Muskelgleichgewichts,

-Wiederherstellung des medialen Stützpunkts des Fußes,

haben unverändert Gültigkeit. Zu erreichen sind diese Ziele über eine metatarsale Korrekturosteotomie in Kombination mit einem periartikulären Weichteileingriff am Großzehengrundgelenk. Um den verschieden stark ausgeprägten Spreizfußfehlstellungen gerecht zu werden, ist es unverzichtbar, mehrere Osteotomieverfahren (distal, diaphysär, proximal) zu beherrschen.

Während die Therapieziele einer Spreizfußkorrektur über viele Jahrzehnte unverändert geblieben sind, haben sich die Ansprüche des Patienten wesentlich verändert. Kosmetische Aspekte der rekonstruktiven Chirurgie am Vorfuß sind ebenso wie die möglichst rasche Rehabilitation bei hohem funktionellen Anspruch in den Vordergrund gerückt. Die frühe funktionelle (gipsverbandfreie) Nachbehandlung ist heute als Nachbehandlungsstandard elektiver Vorfußeingriffe anzusehen.

In diesem Schwerpunktheft sind zwei Operationstechniken für eine distale und eine proximale Osteotomie am ersten Mittelfußstrahl dargestellt. Für die distalen Korrekturosteotomien gilt, dass deren Korrekturmöglichkeit unabhängig vom Design der Osteotomie aufgrund der Breite des distalen Metatarsale begrenzt ist. Bei der Vielzahl diaphysärer und proximaler Korrekturmöglichkeiten erscheint es deshalb weder erforderlich noch sinnvoll, die Indikation für eine distale
Osteotomie über den Grenzbereich von $15^{\circ}$ (erster intermetatarsaler Winkel) auszudehnen.

Die Opening-Wedge-Osteotomie als proximale Korrektur hat in den letzten Jahren an Bedeutung zugenommen. Die Operationstechnik ist jedoch als anspruchsvoll zu bezeichnen, weil der Erhalt der lateralen Kortikalis aus Gründen der Primärstabilität gewährleistet sein muss. Die Stabilisation der aufklappenden Osteotomie hat sich durch die Markteinführung zahlreicher winkelstabiler Implantate deutlich vereinfacht, so dass eine funktionelle Nachbehandlung möglich ist.

Die zusätzliche Korrekturosteotomie der Grundphalanx (Akin-Osteotomie) ist bei korrekter Indikationsstellung eine einfache und wirkungsvolle Operationstechnik. Ähnlich wie bei den metatarsalen Umstellungsoperationen gilt auch hier, dass eine primär übungsstabile Versorgung anzustreben ist, die eine postoperative Mobilisation des Großzehengrundgelenks erlaubt.

Zwei weitere Beiträge dieses Schwerpunkthefts beschäftigen sich mit Operationsverfahren zur Behandlung des Hallux rigidus.

Die Cheilektomie des ersten Mittelfußkopfs in Kombination mit einer extendierenden suprabasalen Osteotomie der Grundphalanx (Operation nach KesselBonney) ermöglicht die Wiederherstellung einer weitgehend ungestörten Roll-Gleit-Bewegung der Grundphalanx und verbessert so die Dorsalextension beim Abrollen des Fußes.

Der "Goldstandard" in der Behandlung des fortgeschrittenen Hallux rigidus besteht unverändert in einer Arthrodese des Großzehengrundgelenks. Wenn jedoch aus unterschiedlichen Gründen (z.B. symptomatische Arthrose des Großzehenendgelenks) die Gelenkbeweglichkeit erhalten bleiben soll, ist die Resektionsarthroplastik nach Valenti aus biomechanischer Sicht der Resektion der Grundphalanxbasis deutlich überlegen, weil die Ansätze der Flexor-hallucis-brevis-Sehne erhalten bleiben. Damit wird die Gelenkstabilität erhalten, und es kommt nicht zur Retraktion der Sesambeine. Zudem sind die Rückzugsmöglichkeiten zu einer Arthrodese günstiger als bei der Grundgliedbasisresektion.

Renée A. Fuhrmann

Oper Orthop Traumatol 2008;20:451

DOI 10.1007/s00064-008-1500-Y 